\title{
A Tunable Capacitor Based on MEMS Technology for RF Applications
}

\author{
Mir Majid Teymoori \\ Department of Electrical Engineering \\ Firoozkooh Branch, Islamic Azad University \\ Firoozkooh, Iran \\ mteymoor@yahoo.com
}

\author{
Jaber Merrikhi Ahangarkolaei \\ Department of Electrical Engineering \\ Firoozkooh Branch, Islamic Azad University \\ Firoozkooh, Iran \\ jmerrikhi@yahoo.com
}

\begin{abstract}
A tunable capacitor based on MEMS technology is presented in this paper. The proposed structure consists of two fixed-fixed parallel movable plates with 4 supports. Movability of two plates makes it possible to actuate the plates with a small voltage. When the actuation is applied, two plates move together and their distance is decreased, hence the capacitance is increased and tuning is achieved. The structure is simulated through the finite element method using COMSOL and ANSOFT HFSS software. Simulation results show that the actuation voltage required to access the $59 \%$ tuning, is $7 \mathrm{~V}$. The effective area of the designed tunable capacitor is $200 \times 200 \mu \mathrm{m} 2$ with a thickness of $2 \mu \mathrm{m}$. The quality factor is 91 at $11 \mathrm{GHz}$ which is sufficient for various RF communication applications such as filters, transceivers and so on. The low stress induced on the plates and low actuation voltage, are the main advantages of the design.
\end{abstract}

Keywords-MEMS; tunable capacitor; fixed-fixed beam; quality factor; finite element method

\section{INTRODUCTION}

The growing demands for the wireless communication systems enforce researchers to design and implement new components required for this purpose. The variable capacitors and variable inductors as main components of a wireless system play a main role and can be employed in frequency based circuits. Diverse applications of he tunable capacitors such as filters, voltage controlled oscillators, super-heterodyne transceivers and so on make them an attractive subject. As technology advances, the quality of tunable capacitors (tuning range, quality factor and broad frequency) increase [1-5].

MEMS (Micro Electro Mechanical Systems) have a very significant impact on the development of tunable devices e.g. varactors. The capability of employing electrostatic actuation and air dielectric makes possible to implement high quality factor and low power consumption through the MEMS technology [6]. There are many methods to design and develop MEMS Variable capacitors. The parallel plate capacitors have been used very well in communication transceivers due to their simple tuning mechanism, low area consumption, high quality factor achievement and MEMS fabrication possibility.
A number of MEMS tunable capacitors have been designed, fabricated and widely studied $[7,8]$. Generally, there are three methods to change the capacitance of the parallel plate capacitors: the gap tuning method [9-12], the area tuning method [13, 14] and the dielectric displacement method [15]. Different actuations are employed in the parallel plate variable capacitors such as electrostatic [16], piezoelectric [17] and thermal actuator [18]. The integrated fabrication compatibility and low power consumption of the electrostatic actuation almost enforce the researchers to employ it. Low actuation voltage and high tuning range are the thermal actuation advantages, however high power consumption is its drawback.

In this paper we present a new low actuation voltage and four-support beam for tunable MEMS capacitors which are studied using FEM software e.g. COMSOL and ANSOFT HFSS. The paper includes two main sections. First section explains the design and analytical considerations, and the second section covers the computer simulation results.

\section{DESIGN AND ANALYTICAL CONSIDERATIONS}

\section{A. Proposed Structure}

Based on the gap tuning method of the parallel plate capacitors, we introduce a new design for the tunable capacitor. As depicted in Figure 1a and Figure 1b, the proposed structure consists of a bottom plate which is located on the etched silicon and can be moved. The upper plate is attached to four supports and is on top of the bottom plate.

The idea of the two plates moving is before introduced in [19] in 2002 for MEMS switches and in [20] for Fixed-Free cantilever beam tunable capacitors. Other researches have also proposed two moveable plate tunable capacitors [21-24]. However, the novelty of the proposed tunable capacitor design is the simultaneous movability of the two plates with an idea to reduce the spring constant of the structure. We employ the fixed-fixed cantilever-section beam which has the smaller spring constant with respect to the fixed-fixed beam counterpart. In our proposed structure, the plate is attached to four supports and hence the stiffness of the plate is reduced considerably. 


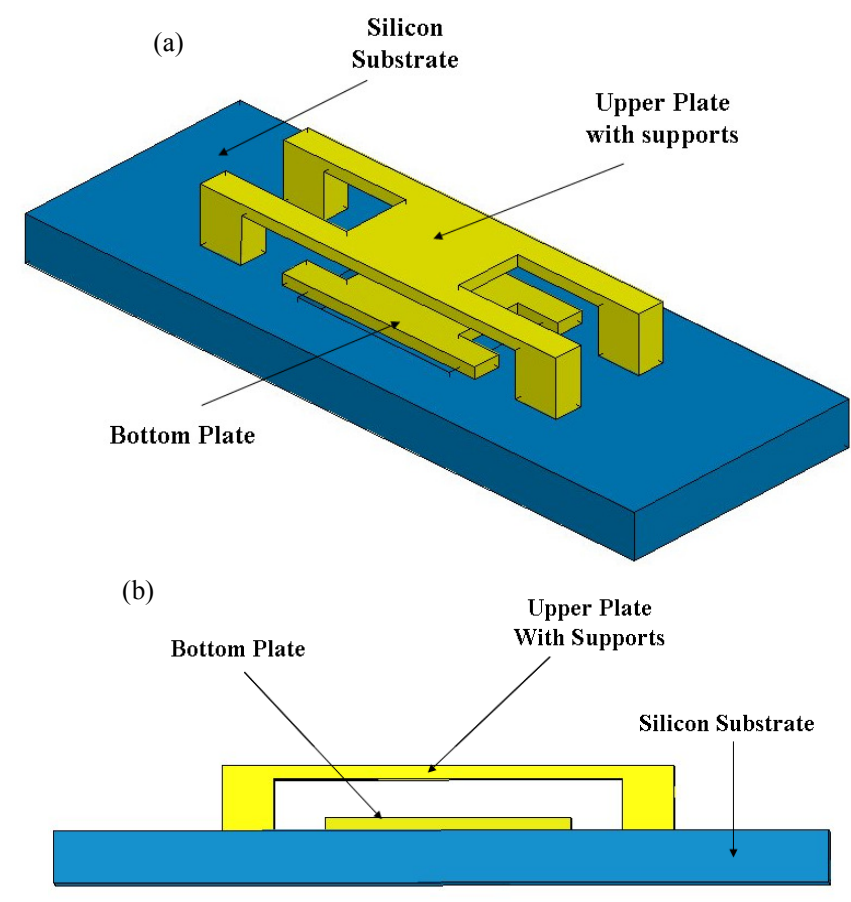

Fig. 1. Schematic of the proposed tunable capacitor: a) isometric view and b) front view

\section{B. Tuning Mechanism}

When the voltage is applied between the bottom and upper plate, the two plates start to move together toward each other and the gap between them $(d)$ is decreased and regarding to (1) the capacitance change is achieved.

$$
C=\frac{\varepsilon_{0} \varepsilon_{r} A}{d}
$$

Assuming the gap between two plates $d$, the structure is designed so that each plate displaces only $d / 6$ and total displacement is $d / 3$. The one-third displacement has three main advantages: the first it prevents the pull-in effect of the electrostatic actuation and makes it possible to have good control on the capacitance change. The second benefit is the structure's stress and strain reduction, which increases the lifetime of the designed capacitor. Third advantage is the linearity behavior of the capacitance change in the one-third of gap between two plates.
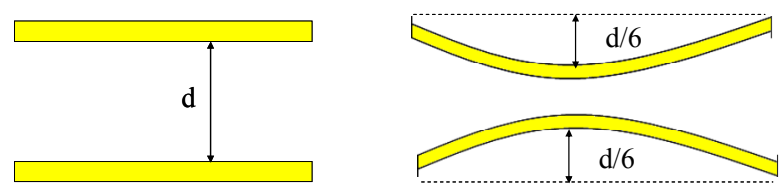

Fig. 2. Tuning mechanism of the designed tunable capacitor

\section{Analytical Considerations}

1) Structure Stiffness

The designed plates for the tunable plate can move simultaneously. Referring to Figure $3 a$, the relationship between the electrostatic force induced by the voltage applied between the two plates and the plate displacement is expressed as:

$$
\begin{aligned}
& F=K_{1}\left|X_{0}-X_{1}\right| \\
& F=K_{2}\left|X_{3}-X_{2}\right|
\end{aligned}
$$

Where $K_{1}$ and $K_{2}$ are the spring constant of the up and bottom plates, respectively. For Figure $3 b$, we would have:

$$
F=K\left|X_{0}-X_{4}\right|
$$

Where, $K$ is spring constant of the upper plate individually and $F$ is the same as the Figure 3a. Considering Figure 3:

$$
X_{0}-X_{4}=\left(X_{0}-X_{1}\right)+(X 2-X 3)
$$

Combining (3), (4) and (5):

$$
\frac{F}{K}=\frac{F}{K_{1}}+\frac{F}{K_{2}}
$$

If two plates have the same configurations and the same boundary conditions, then $K_{l}=K_{2}$ and hence $K=K_{l} / 2$, that is the spring constant of the total structure is the half of the one plate moving structure and therefore the electrostatic voltage will be small. Regarding this phenomena, to achieve the low actuation voltage we choose two movable plates for our design.
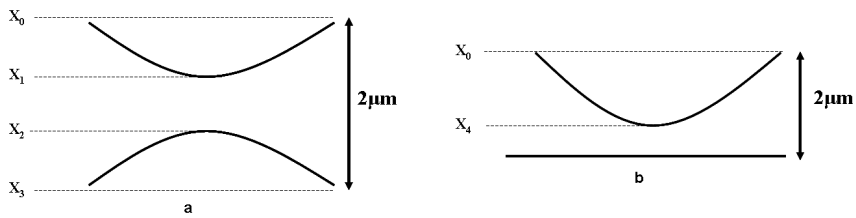

Fig. 3. Comparison of two plates: a) two plates moving b) only one plate moving

According to Euler-Bernoulli Equation, The governing equation for bending of the isotropic beams of constant cross section is as [25]:

$$
E I \frac{d^{4} w(x)}{d x^{4}}=q(x)
$$

Where $E$ is the young's modulus, $I$ is the moment of inertia, $w(x)$ is the deflection point and $\mathrm{q}(\mathrm{x})$ is the applied transverse load. For a fixed-fixed plate, assuming no residual stress, the spring constant can be approximately calculated as [26]:

$$
K=16 E w\left(\frac{t}{l}\right)^{3}
$$

Where $w, t$ and $l$ is the width, thickness and length of the plate, respectively. Equation (8) is true for two sides completely fixed plate. In our designed plate, we reduce the 
boundary condition employing four supports, hence the stiffness and the applied voltage is diminished considerably.

\section{2) Pull-in Voltage}

When the voltage is applied between two parallel plates, the plates start to deflect downward, decreasing the gap $g$ and increasing the electrostatic pressure on the plates. The governing equation is:

$$
m g^{\prime \prime}+b g^{\prime}+k\left(g-g_{0}\right)=F_{e}=\frac{\varepsilon A V^{2}}{2\left(g+\frac{t_{d}}{\varepsilon_{r}}\right)^{2}}
$$

Where $m$ is the mass, $b$ is damping coefficient and $k$ is the stiffness of the plate, $g_{0}$ is the gap between two plates, $A$ is the plate's effective area, $t_{d}$ is the thickness of the dielectric, $\varepsilon$ is the air permittivity, $F_{e}$ is the electrostatic force and $V$ is the applied voltage. Neglecting the mass and damping force, (9) has a critical point at $g=(2 / 3) \cdot g_{0}+t_{d}$. Replacing this value in (9), we have [27]:

$$
V_{P}=\sqrt{\frac{8 K d^{3}}{27 \varepsilon_{0} A}}
$$

This voltage $\left(V_{p}\right)$ is known as the pull-in voltage and is a critical voltage for two plates contacting. Equation 10 shows that when the distance between the two plates is decreased, the actuation voltage can be reduced.

\section{SimUlation}

To evaluate the actuation voltage and frequency response of the designed tunable capacitor, the structure is simulated employing the finite element method with COMSOL and ANSOFT HFSS software. The COMSOL software is used to find the required voltage for one-third displacement of two plates and the HFSS software gives us the frequency characteristics of variable capacitor such as quality factor and tuning ratio.

\section{A. Pull-In voltage Simulations}

Two structures are considered in the COMSOL software simulation: one structure is a two clamped membrane with size of $200 \times 200 \times 2 \mu \mathrm{m}^{3}$ (Figure 4a) and the other is our designed structure (Figure $4 b$ ).

The main aim of this simulation is to compare the two structures with varying stiffness (different spring constants $K$ ). To simplify, in all simulations it is assumed that the residual stress on the membrane is zero. It is evident that residual stress will increase the actuation voltage. The mechanical properties of the beam material (gold) are stated in Table I for this simulation.

Based on the simulation results, the pull-in voltage for the structure shown in Figure $4 \mathrm{a}$ is approximately $64 \mathrm{~V}$. The displacement and stress distribution of this simulation is shown in Figures 5 and 6, respectively. As shown in Figure 6, the maximum stress on this structure is $23.6 \mathrm{MPa}$ which is much smaller than the critical stress of gold (205 MPa).

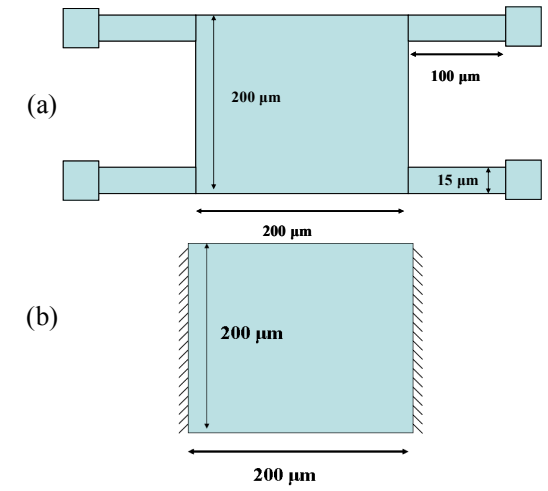

Fig. 4. Schematic of the simulated beams: a) our designed beam b) two clamped beam

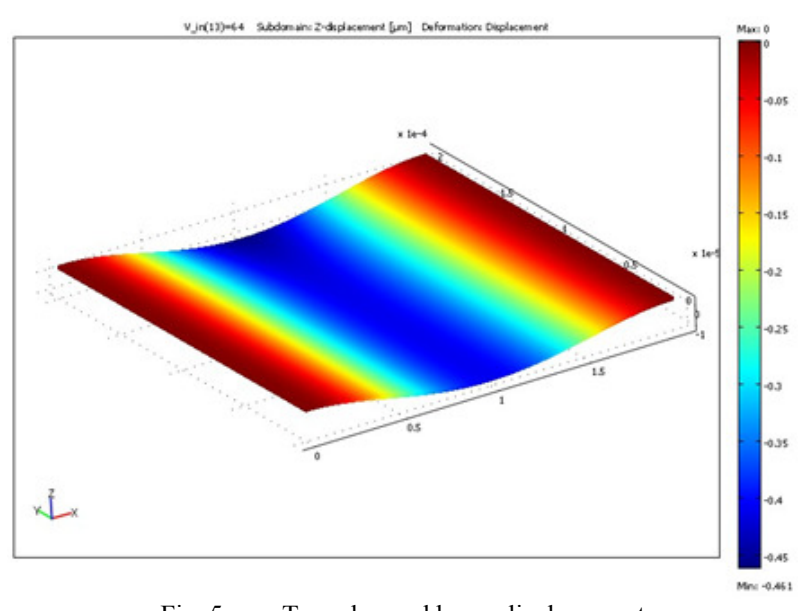

Fig. 5. Two clamped beam displacement

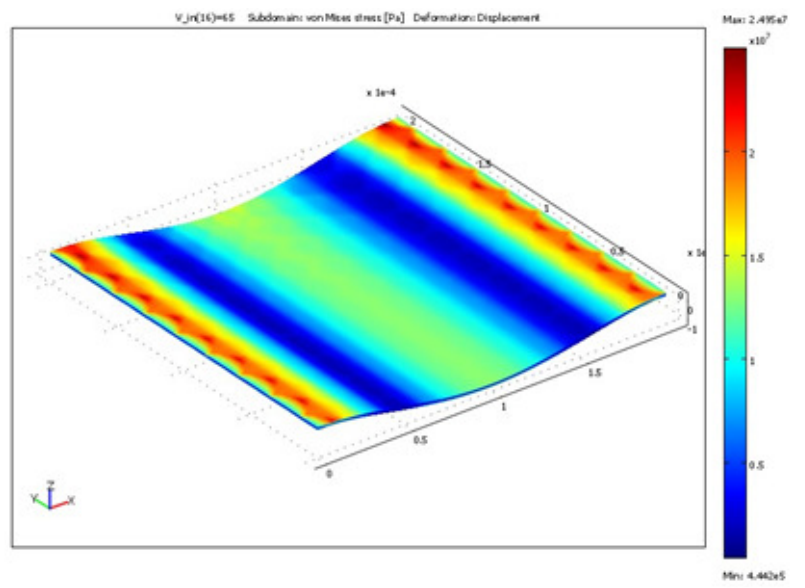

Fig. 6. Total stress distribution on the two clamped beam

For the proposed structure shown in Figure $4 \mathrm{~b}$, the same simulation is accomplished and the displacement and total stress distribution on the beam is depicted in Figures 7 and 8 , respectively. It should be mentioned that the deformation in both figures was exaggerated for visualization purposes, so the bottom plate extends in the area of the upper plate and vice versa. 
Based on Figure 8, the stress on the designed structure is 7.1 $\mathrm{MPa}$ which is almost 3 times smaller than the Figure $4 \mathrm{a}$ structure and very smaller than the gold material critical stress, hence the lifetime of the proposed structure can be very good. As shown in Figure 7, the actuation voltage (for one-third displacement) in this structure is approximately $7 \mathrm{~V}$ which is the main advantage of the 4-support beam structure.

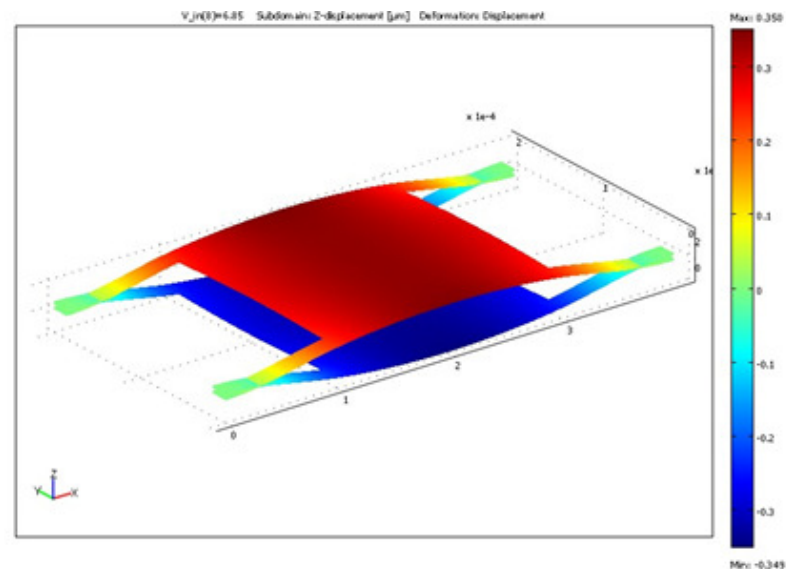

Fig. 7. Four-support beam displacement

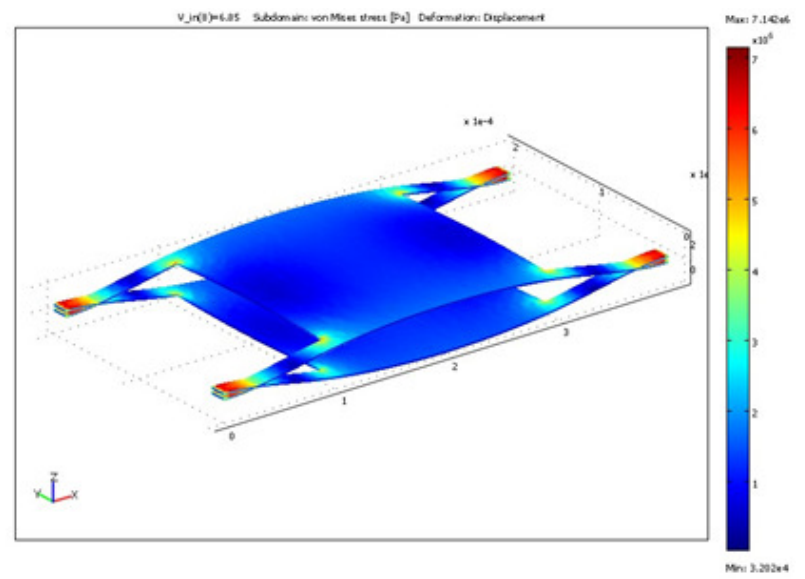

Fig. 8. Stress distribution on the proposed four-support beam

\section{B. Frequency Simulation}

To evaluate the frequency behavior of the designed capacitor, we employ the ANSOFT HFSS software which is very suitable for the RF applications. Table 2 shows the electrical properties of the designed beam material. The main aim of this simulation is to find the capacitance ratio (tuning range) and the quality factor.

The HFSS simulation results are shown in the Figures 9 and 10. As depicted in the Figure 9, the capacitance is changed from $312 \mathrm{fF}$ to $497 \mathrm{fF}$ in the frequency of $11 \mathrm{GHz}$; hence we would have $59 \%$ tuning range. In This frequency, the quality factor is 90 , which is very suitable for RF communication applications.

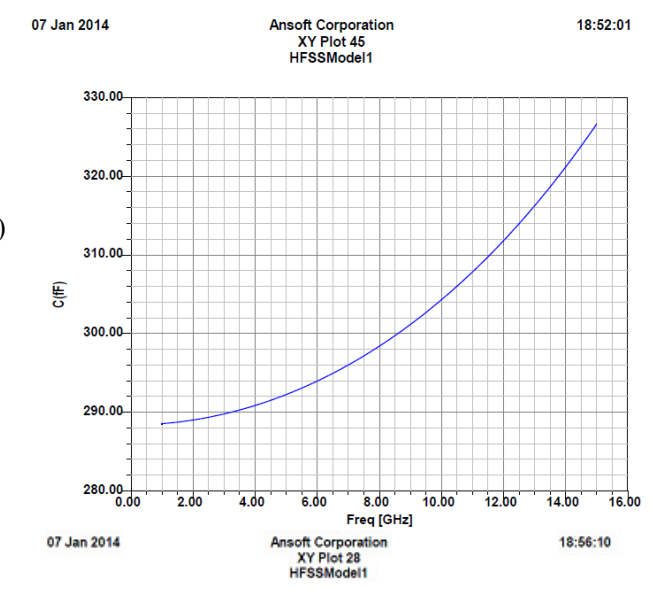

(b)

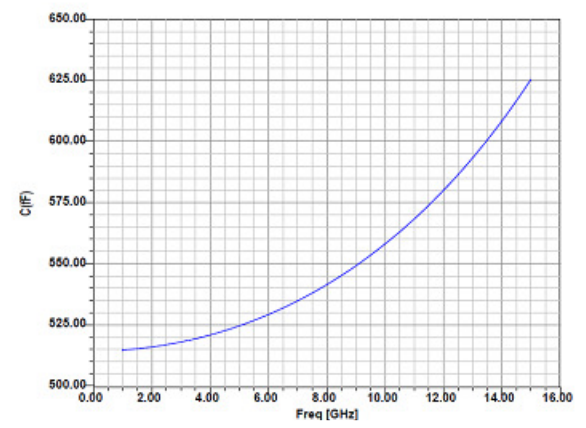

Fig. 9. The capacitance of the proposed varactor a) before and b) after actuation

(a)

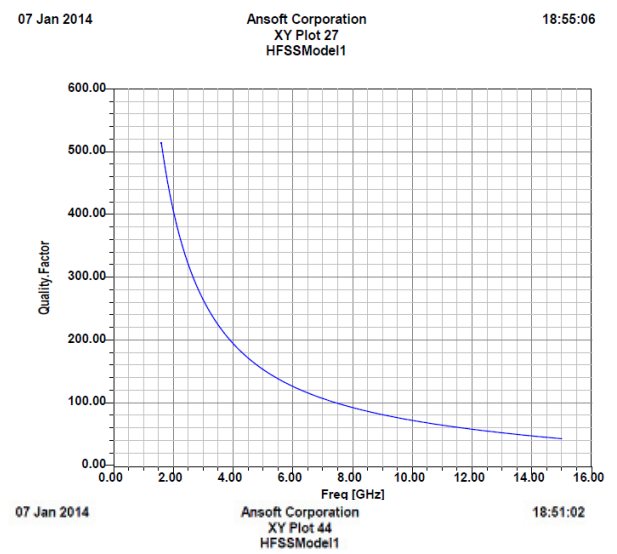

(b)

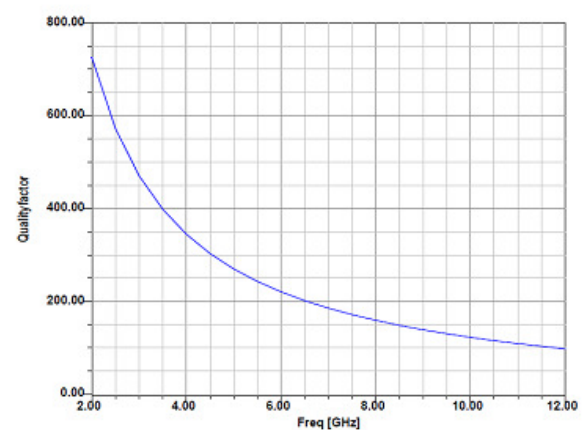

Fig. 10. The Quality factor of the proposed varactor a) before and b) after actuation 
TABLE I. MECHANICAL PROPERTIES OF BEAM MATERIAL (GOLD)

\begin{tabular}{|c|c|}
\hline Parameter & Value \\
\hline Yong's Modulus & $76.52 \mathrm{GPa}$ \\
\hline Poisson Ratio & 0.41 \\
\hline Density & $19300 \mathrm{~kg} / \mathrm{m}^{3}$ \\
\hline Yield stress & $205 \mathrm{MPa}$ \\
\hline Area & $200 \times \mathrm{m}^{2}$ \\
\hline Thickness & $2 \mu \mathrm{m}$ \\
\hline Gap & $2 \mu \mathrm{m}$ \\
\hline Support Length & $100 \mu \mathrm{m}$ \\
\hline Support Width & $15 \mu \mathrm{m}$ \\
\hline
\end{tabular}

TABLE II. ELECTRICAL PROPERTIES OF THE USED MATERIAL

\begin{tabular}{|c|c|c|}
\hline Material & Conductivity & Relative Permittivity \\
\hline Silicon Substrate & $0.003 \mathrm{~S} / \mathrm{m}$ & 11.9 \\
\hline Silicon Nitride & $0.001 \mathrm{~S} / \mathrm{m}$ & 7 \\
\hline Gold & $41 \mathrm{e} 6 \mathrm{~S} / \mathrm{m}$ & 1 \\
\hline Air & 0 & 1.0006 \\
\hline
\end{tabular}

\section{CONCLUSION}

A low voltage tunable capacitor based on micromachining technology is designed and simulated. The tuning mechanism is based on the gap tuning of a new structure. Two plates move-ability design decreases the stiffness of the total structure and hence the applied voltage is considerably decreased. The structure is simulated with FEM COMSOL and ANSOFT HFSS software. The results show $7 \mathrm{~V}$ applied voltage for deflection of the $0.35 \mu \mathrm{m}$ of plates individually and a $59 \%$ tuning range with quality factor of 90 at $11 \mathrm{GHz}$ which is compatible with the RF applications.

\section{ACKNOWLEDGMENT}

The authors would like to thank the Department of Electrical Engineering, Firoozkooh branch, Islamic Azad University, Firoozkooh, Iran for the financial support.

\section{REFERENCES}

[1] H. L. Krauss, C. W. Bostian, F. H. Raab, Solid State Radio Engineering, New York, NY, USA: John Wiley \& Sons, 1980.

[2] G. M. Rebeiz, RF MEMS theory, design and Applications. New Jersey, USA: John Wiley \& Sons, 2003.

[3] J. J. Yao, "RF MEMS from a device perspective", J. Micromech. Microeng., Vol. 10, pp. 9-38, 2000

[4] H. J. De Los Santos, RF MEMS Circuit Design for Wireless Communications, Artech House, 2002

[5] D. J. Young, B. E. A. Boser, "Micromachined Variable Capacitor for Monolithic Low-Noise VCOs", Solid-State Sensor and Actuator Workshop, Hilton Head, pp.86-89, USA, 1996

[6] W. Wan, D. Lowther, "Design and synthesis of wide tuning range variable comb drive MEMS capacitors", COMPEL, Vol. 26, pp. 689699, 2006

[7] M. Dong, J. Xiang, P. H. Wang, Z. Young, X. L. Zhao, "Fabrication and dynamic analysis of the electrostatically actuated MEMS variable capacitor”, Microsyst. Technol., Vol. 14, pp. 397-402, 2008

[8] H. Mahmoodnia, B. A. Ganji, "A Novel High Tuning ratio MEMS Cantilever Variable Capacitor”, Microsyst. Technol., Vol. 19, pp. 19131918,2013
[9] A. Dec, K. Suyama, "Micromachined electro-mechanically tunable capacitors and their applications to RF IC's", IEEE T. Microw. Theory, Vol. 46, pp. 2587-2595, 1998

[10] H. Nabovati, K. Mafinejad, H. Keshmiri, A. Nabovati, "Design and Simulation of an Oblique Suspender MEMS Variable Capacitor", SCI IRAN, Vol. 13, pp. 60-66, 2006

[11] J. Zou, C. Liu, J. Schutt-Aine, "Development of a wide tuning-range two-parallel-plate tunable capacitor for integrated wireless communication systems", International Journal of RF and Microwave Computer-Aided Engineering, , Vol. 11, pp. 322-329, 2001

[12] L. Dussopt, G. M. Rebeiz, "High-Q millimeter-wave MEMS varactor: Extended tuning range and discrete-position designs", IEEE MTT-S International Microwave Symposium Digest, Seattle, WA, IEEE. pp. 1205-1208, USA, 2002

[13] A. Oz, CMOS/BiCMOS self-assembling and electrothermal microactuators for tunable capacitors, MSc, Carnegie Mellon University, Pittsburgh, USA, 2003

[14] J. J. Yao, S. T. Park, J. DeNatale, "High tuning-ratio MEMS-based tunable capacitors for RF communication applications", Solid-State and Actuator Workshop, Hilton Head, pp. 124-127, USA, 1998

[15] J. J. Yao, S. T. Park, R. Anderson, J. DeNatale, “A low power/low voltage electrostatic actuator for RF MEMS applications", Solid-State Sensors and Actuator Workshop, Hilton Head, pp. 246-249, USA, 2000

[16] C. L. Goldsmith, A. Malczewski, Z. J. Yao, S. Chen, J. Ehmke, D. H. Hinzel, "RF MEMS variable capacitors for tunable filters", International Journal of RF and Microwave Computer-Aided Engineering, Vol. 9, pp. 362-374, 1999

[17] J. Y. Park, Y. J. Yee, H. J. Nam, J. U. Bu, "Micromachined RF MEMS tunable capacitors using piezoelectric actuators", EEE MTT-S International Microwave Symposium Digest, pp. 2111-2114, 2001

[18] Z. Feng, H. Zhang, W. Zhang, B. Su, K. C. Gupta, V. M. Bright, Y. C. Lee, "MEMS based variable capacitor for millimeter-wave applications", Solid-State Sensors and Actuators Workshop, pp. 255258,2000

[19] S. Afrang, E. Abbaspour-Sani, “A Low Voltage Capacitive Micromachined Microwave Switch", IEEE International Conference On Semiconductor Electronics, Kualalampur, pp. 110-114, Malaysia, 2002

[20] E. Abbaspour-Sani, N. Nasirzadeh, G. Dadashzadeh, "Two Novel Structures for Tunable MEMS Capacitor with RF Applications", Progress in Electromagnetic Research, Vol. 68, pp. 169-183, 2007

[21] L. Xiuhan, Y. Xia, L. Jian, L. Yin, Y. Liu, D. Fang, H. Zhang, "Tunable RF MEMS capacitor for wireless communication", International Conference on Optical Instruments and Technology, China, October 1922, 2009

[22] Y. Kim, S. G. Lee, S. Park, "Design of the movable plate type MEMS voltage tunable capacitor", International Conference on Modeling and Simulation of Microsystems, pp. 246-249, 2002

[23] B. Kaseem, Masher, R. M. Raafar, "Two movable plate nitride loaded MEMS variable capacitor", IEEE T Microw. Theory, Vol. 52, pp. 831837,2004

[24] Y. Kim, S. Park, "Design of the RF MEMS voltage tunable capacitor for wide tunable range", International Symposium on Microelectronics and MEMS, Adelaide, Australia, 2001

[25] J. M. Gere, S. P. Timoshenko, Mechanics of Materials, PWS Publishing Company, 2003

[26] S. Afrang, G. Rezazadeh, "Design and simulation of simple and varying section cantilever and fixed-fixed end types MEMS switches", IEEE International Conference On Semiconductor Electronics, Kualalampur, pp. 593-596, Malaysia, 2004

[27] M. M. Teymoori, Design and Simulation of a Novel Electrostatic Micromachined pump for Medical Applications, MSc, Urmia University, Iran, 2003 